\title{
Sensor Technologies for Prognostics and Health Management of Electronics
}

\author{
Wenxue Yang1,a, Zhe Chen ${ }^{2,3, b}$ and Feng Yang ${ }^{3, c}$ \\ ${ }^{1}$ School of Electron and Information, Hubei Three Gorges Polytechnic, Yichang, Hubei, P. R. China \\ ${ }^{2}$ Computer school, Wuhan University, Wuhan, Hubei, P. R. China \\ ${ }^{3}$ School of Computer Science, China University of Geosciences, Wuhan, Hubei, P. R. China \\ ayangwx@xinhuanet.com, bwdchenzhe@163.com, 'cqwioercug@sina.com
}

Keywords: sensor, prognostics and health management, electronics, sensor development

\begin{abstract}
This paper introduces the sensors and their sensor technologies. The required attributes of sensors for the development for PHM of electronics are discussed. Finally, their trends in sensor systems are presented.
\end{abstract}

\section{Introduction}

Electronics constitute a major component of our industrial systems. They are the dominant element in some areas such as communications, computing, control, etc. Prognostics and Health Management (PHM) major in how sensor technologies are monitoring electronics systems [1]. Sensors and sensing strategies constitute the foundational basis for fault diagnosis and prognostics. Sensors provide the means for us by which this signal can be acquired, processed, and stored.

A class of PHM sensors that finds significant inroads into the application domain includes accelerometers eddy-current ultrasonic sensors, micro-electro mechanical systems (MEMS) and fiber-optics. We will focus on a few typical and innovative sensors that are finding widespread use or promise to expand their utilities in PHM for the electronics.

\section{Sensor and sensing technologies}

A sensor is defined as a device that provides a usable output signal in response to a specified measurement. A sensor also translates physical, chemical, and biological phenomena into the electronic signals by their mechanisation. A sensor is also defined as a device that is sensitive to light, temperature, electrical impedance, or radiation level and transmits a signal to a measuring or control device. Sensor system is very complex. Sensor hardware for PHM applications relate to the utility of smart sensors and transducers, MEMS, networking and interfacing, DSP-based multitasking, and multiprocessing platforms for the efficient, reliable, and cost-effective implementation of software routines. These sensor systems are placed almost exclusively to interrogate and track system properties that are related directly to their failure mechanisms.

Some electrical parameters, for example, voltage, current, power and frequency, are usually examined in PHM of electronics. There are commonly used in voltage measurement inductive, thermal, capacitive, and Hall-effect sensors.

One method of current measurement is by Hall effect sensors. Another method of current measurement is the current-to-voltage conversion which is based on Ohm's law. The voltage may be measured by digital meters and oscilloscopes.

Power is the production of the current and the voltage. Power sensor always includes an analog multiplier and a voltage-current converter. So the power may be measured by indirect approach.

It is to measure frequency that depends on the number of occurrences of a repeating event per unit time. A measuring frequency approach is to use timer, which accumulates the number of event in a period time. Before to the multiplier, signal should pass the amplifier as well as filtering to suitable for counting. 
The most widely used thermal sensors are RTDs, thermocouples, and semiconductor junction diodes. RTDs may operate on the principle in which the electrical resistance of the sensor changes predictably with temperature in a repeatable manner. Unlike the basic RTDs, it may be molded or compressed into various shapes to fit a wide of applications.

Ultrasonic waves can travel long distances and thus have the potential to monitor a large volume of material, because ultrasonic methods have proven useful for nondestructive inspection (NDI) [2].

Magnetic sensors mainly include Hall effect sensor, magnetoresisittive sensor, magnetometers, magnetotransistor, magnetodiode, and magneto-optic sensor. Magnetotransistor is the property of a material to change the value of its electrical resistance when an external magnetic field is applied for it. Magnetotransistor sensor always comes in a bridge configuration with four magnetically sensitive resisitors in a Wheatstone bridge configuration. In the presence of a magnetic field, the values of the resistors change, causing a bridge imbalance and generating an output voltage proportional to the magnetic field strength.

Micro-electro mechanical systems (MEMS) sensors based on fiber-optic technologies are finding popularity because of their size, cost, and ability to integrate multiple transducers into a single device. MEMS devices in silicon or other materials are fabricated in a batch process with the potential for integration with electronics, thus facilitating on-board signal processing and other 'smart' functions. They have been manufactured in the laboratory or are available commercially, monitoring such critical parameters as temperature, pressure, acceleration.

\section{Sensor localization}

Localization of sensors to monitor fault signatures accurately and robustly in a critical military or industrial system constitutes an essential function of the PHM design process. It is instructive to take advantage of such sensors in a fault diagnosis monitoring scheme because they can provide useful information relating to fault behaviors of critical system variables.

Four main requirements must be met to place sensors optimally for fault diagnostic purposes: detectability, identifyability, fault-detection reliability, and a requirement associated with limited resources.

Typical devices in this class are accelerometers for vibration monitoring, proximity sensors, etc. More recently, research on sensor placement has focused on two different levels: the component level and the system level. At the electrical component level, attempts have been reported by regarding placement at the component's range. For complex, large-scale systems consisting of multiple subsystems, a fault may propagate through several components. With a large number of possible sensor locations, selection of an optimal location, as well as the number and types of sensors, poses a challenging problem that must be addressed at the system level.

We can achieve a particle-swarm optimization method where sensors are selected for every fault on the basis of a heuristic search algorithm. To estimate the fault-detecting error rate is the objective of performance-assessment module.

\section{Sensors development for PHM}

The requirements of sensor system for PHM depend on our specific application, but there are some considerations. The PHM of electronics sensor system will have sensors, onboard analog-to-digital converters (ADC), memory, embedded chips, dada transmission, and the power source, and so on. That is shown in Fig 1. But every sensor system will not contain all above elements. So we should considerate sensor system on selecting PHM applications of electronics. 


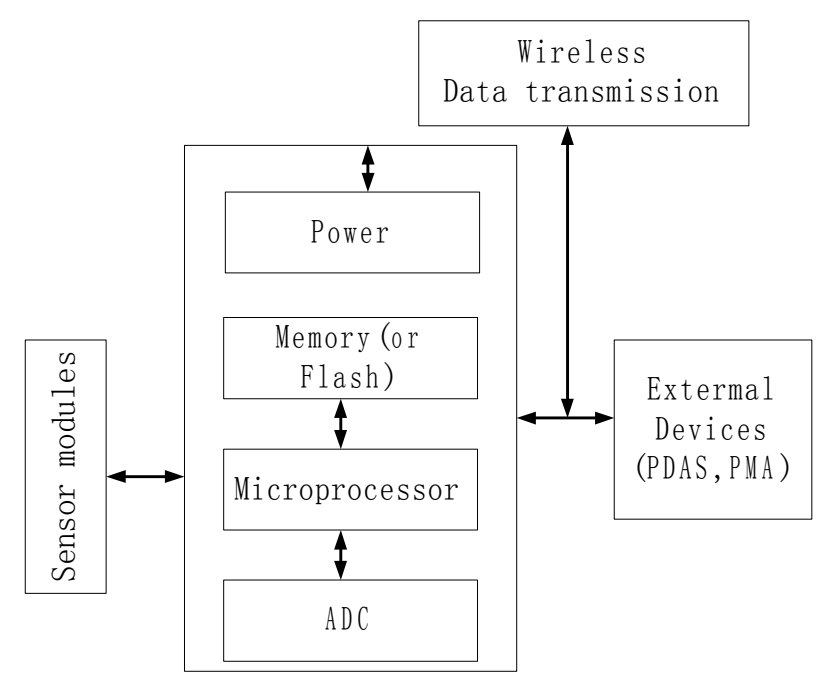

Fig. 1 sensors development for PHM of electronics

We may considerate the two aspects, parameters of monitoring and performance of sensor system. Parameters of monitoring systems can be selected based on their relationship to function that are likely to implicated in failures. Selection should be based on the data and knowledge of critical parameters. More systematic methods, such as FMECA, can be used to determine parameters than need to be monitored. The required performance should be considered during the analysis of the application. The relevant performance include: functional attributes of sensor systems, onboard power and management, sampling mode and sampling rate, fast and convenient data transmission, reliability, etc. The sensor systems to PHM of electronics may be very advanced. Smart sensors and Wireless sensor networks (WSNs) will be developed. The following describes their approach and their implementation into PHM of electronics.

\section{Smart sensors}

Smart sensors are one of the essential components of PHM. Smart sensors are one of basic sensing elements with embedded intelligence, capable of networking among themselves and with higher-level systems to provide both process data and data validity qualifiers to assess the health of electronic systems [3].

Smart sensors allow a PHM architecture that relies on acquiring information from smart sensors, processing this information, comparing the information provided by the sensors' embedded knowledge to its own knowledge information system, and establishing the health of the system, estimating the remain useful life. With the IEEE 1451 guidelines, sensor systems can: provide accurate and reliable data to the user; conduct sensor and system health checks; communicate with other sensor suites to validate the data and health of the sensor(s); contain sensor identification and characterization parameters; contain parameters to perform sensor validation and measurement interpretation; and to provide sensor data synchronization.

Because smart sensors are embedded intelligence and smart algorithms such as self-calibration, self-health assessment, self-healing, and preprocessing of raw data at the sensor level, which will provide for a more reliable and robust system. So smart sensors can be form PHM of electronics capable of predicting the near-term and long-term health issues of the system being monitored.

\section{Wireless sensor networks}

Wireless sensor networks (WSNs) are important in PHM applications. Properly designed WSNs can be installed and calibrated quickly and can be up and running in a very short time frame. WSNs generally consist of a data-acquisition network and a data-distribution network monitored and controlled by a management center. The plenty of available technologies makes even the selection of components difficult, let alone the design of a consistent, reliable, robust overall system. The study of 
WSNs is challenging in that it requires an enormous breadth of knowledge from a large variety of disciplines.

WSNs consist of multiple sensor nodes that are capable of communication with each other and collaborating on a common sensing goal. The advantage of WSNs is that they allow data from multiple sensors to be combined or fused to obtain inferences. This is referred to as multi-sensor data fusion. A communication network is composed of nodes, each of which has computing power and can transmit and receive messages over communication links, wireless or cabled. The network topologies may include fully connected, mesh, star, ring, tree, and bus.

The hoc networks of geographically distributed sensors in remote environments can apply in PHM of electronics. Meanwhile, software power management techniques can decrease the power consumed by RF sensor nodes significantly.

\section{Trends in sensor technologies for PHM of Electronics}

The single smart sensor is migrating toward multi-sensor systems aided by miniaturization, dramatic cost reductions, wireless technologies, and networking [4]. Networked embedded sensor systems are to improve our monitoring capabilities significantly in large distributed systems and increase the accuracy and reliability of measurements through multi-sensor fusion, advanced data mining, and other intelligent techniques.

Ultra-low-power electronics enable sensor system to consume much lower, for the example the CPU chips of MSP430S149 and C8051F039. With the development of new materials and energy technologies, battery-free sensor system will be to consider, especially for use in embedded, remote, and other monitoring conditions.

The development of wireless transmission technology will be to long-distance, high precision, and more future sensor system. In future nodes of smart sensor will be highly intelligent, with very more functions. A new generation of sensors is envisioned through incorporating embedded intelligence. Smart Algorithms such as self-calibration, self-health assessment, self-healing, and preprocessing of raw data at the sensor level, will provide for a more reliable and robust system. They will have the capabilities of built-in-test diagnostics and prognostics, which will make the wireless sensor network more advanced. These smart sensors will form PHM of electronic systems capable of predicting the near-term and long-term health issues.

\section{References}

[1] N. Vichare and M. Pecht, "Prognostics and Health Management of Electronics", IEEE Transactions on Components and Packaging Technologies. Vol.29.NO.1, pp.222-229, March, 2006.

[2] K.R.Leonard, E.V.Malyarenko, and M. K. Hinkers, "Ultrasonic Lamb Wave Tomography," Inverse Problems 18(6): 1795-1808,2000.

[3] B.Betts,"Smart Sensors", IEEE Spectrum Magazine, VOL43, NO. 4, pp.50-53, April 2006.

[4] O.Kanoun and H. Trankler, "Sensor Technology Advances and Future Trends", IEEE Transactions on Instrumentation and Measurement 53(6):1497-1501, 2004.

\section{Acknowledgments}

The authors would like to thank all colleagues who previously provided technical support. 